\title{
INSULIN CARDIOPLEGIA FOR ELECTIVE CORONARY BYPASS SURGERY
}

Vivek Rao MD, PhD

Michael A. Borger, MD

Richard D. Weisel, MD

Joan Ivanov, RN

George T. Christakis, MD

Gideon Cohen, MD

Terrence M. Yau, MD

For the Insulin Cardioplegia Trial (ICT) Investigators* $^{*}$
Background: Improved methods of myocardial preservation are required to reduce the morbidity and mortality of coronary bypass surgery for high-risk subgroups. Metabolic stimulation with insulin, glucose solutions, or both has been proposed as a method to preserve the ischemic myocardium. We performed a prospective, double-blind, randomized trial to evaluate the effects of insulin and glucose as cardioplegic additives when used as part of a tepid continuous blood cardioplegic strategy.

Methods: We randomized 56 male patients undergoing elective isolated coronary bypass surgery to 1 of 4 cardioplegic groups containing either 42 or 84 $\mathrm{mmol} / \mathrm{L}$ glucose with or without $10 \mathrm{IU} / \mathrm{L}$ of insulin. Perioperative assessments of myocardial metabolism and left ventricular function were performed.

Results: Insulin-enhanced cardioplegia was associated with beneficial effects on both myocardial metabolic and functional recovery after cardioplegic arrest. Insulin's effect was independent of the ambient glucose concentration.

Conclusions: Cardioplegic formulations containing a $42 \mathrm{mmol} / \mathrm{L}$ concentration of glucose and a $10 \mathrm{IU} / \mathrm{L}$ concentration of insulin provide significant benefit to patients undergoing isolated coronary bypass surgery. The clinical effect of these formulations will need to be assessed in high-risk subgroups of patients, such as those with unstable angina, recent myocardial infarction, or poor left ventricular function. (J Thorac Cardiovasc Surg 2000;119:1176-84)
$T^{1}$ he contemporary results of coronary artery bypass grafting are excellent with low overall morbidity and mortality. ${ }^{1}$ However, as percutaneous ${ }^{2}$ and minimally invasive $^{3}$ approaches to the surgical treatment of coronary artery disease become more common, the demographic profile of patients requiring conventional surgical revascularization will change. We have already documented an increasing proportion of high-risk

From The Toronto General Hospital and Sunnybrook Health Science Centre at The University of Toronto, Toronto, Ontario, Canada.

Supported in part by the Heart and Stroke Foundation of Canada (grants NA3767 and NA4189) and the Medical Research Council of Canada (grant MT13513). V.R., M.A.B., and G.C. are Research Fellows of the Heart and Stroke Foundation of Canada. T.M.Y. is a Research Fellow of the Medical Research Council.

Received for publication Sept 3, 1999; revisions requested Oct 28, 1999; revisions received Dec 7, 1999; accepted for publication Dec 20, 1999.

Address for reprints: Richard D. Weisel, MD, Chair, Division of Cardiovascular Surgery, EN 14-215, The Toronto Hospital, 200 Elizabeth St, Toronto, Ontario, Canada M5G 2C4.

*For a listing of the Insulin Cardioplegia Trial (ICT) Investigators, see the appendix.

Copyright $@ 2000$ by The American Association for Thoracic Surgery 0022-5223/2000 $\$ 12.00+0 \quad \mathbf{1 2 / 1 / 1 0 5 2 5 7}$

doi: $10.1067 / \mathrm{mtc} .2000 .105257$ patients admitted for coronary bypass surgery, valvular surgery, or both at our institution. ${ }^{4,5}$ These patients often have poor preoperative left ventricular function, ${ }^{6}$ unstable angina, ${ }^{7}$ or advanced age ${ }^{8}$ and pose a challenge for intraoperative myocardial protection. Therefore improved methods of cardioplegic protection are required to lower the risks of surgery for these subgroups of patients.

Glucose-insulin-potassium solutions have been commonly used to treat ischemic myocardium in a variety of medical and surgical settings. ${ }^{9,10}$ Unfortunately, the results of earlier investigations were often conflicting, and interest in metabolic stimulation of the heart waned. One potential confounding factor in the early studies of glucose-insulin-potassium solutions was the routine use of moderate hypothermic $\left(25^{\circ} \mathrm{C}-28^{\circ} \mathrm{C}\right)$ cardiopulmonary bypass. In retrospect, metabolic stimulation of the heart may not have been effective at temperatures that inhibit normal enzyme function. The advent of normothermic or warm heart surgery ${ }^{11}$ prompted renewed interest in myocardial protection. We believe that a reassessment of myocardial metabolic stimulation is required at normothermic temperatures, which more 
Table I. Preoperative patient characteristics

\begin{tabular}{|c|c|c|c|c|c|}
\hline & Group $1(n=10)$ & Group $2(n=12)$ & Group $3(n=17)$ & Group $4(n=17)$ & $\mathrm{P}$ value \\
\hline Age (y) & $60 \pm 8$ & $64 \pm 8$ & $60 \pm 9$ & $60 \pm 8$ & .54 \\
\hline \multicolumn{6}{|l|}{ LVEF } \\
\hline$>60 \%$ & $3(30 \%)$ & $1(8 \%)$ & $6(35 \%)$ & $8(47 \%)$ & .25 \\
\hline $40 \%-59 \%$ & $7(70 \%)$ & $11(92 \%)$ & $11(65 \%)$ & $8(47 \%)$ & \\
\hline $20 \%-39 \%$ & $0(0 \%)$ & $0(0 \%)$ & $0(0 \%)$ & $1(6 \%)$ & \\
\hline Triple vessel disease & $8(80 \%)$ & $12(100 \%)$ & $14(82 \%)$ & $14(82 \%)$ & .40 \\
\hline Left main disease & $0(0 \%)$ & $3(25 \%)$ & $2(12 \%)$ & $1(6 \%)$ & .24 \\
\hline PVD & $0(\%)$ & $1(8 \%)$ & $1(6 \%)$ & $0(0 \%)$ & .56 \\
\hline Hypertension & $6(60 \%)$ & $7(58 \%)$ & $7(41 \%)$ & $7(41 \%)$ & .63 \\
\hline
\end{tabular}

Group 1, $42 \mathrm{mmol} / \mathrm{L}$ glucose and no insulin; group 2, $42 \mathrm{mmol} / \mathrm{L}$ glucose and $10 \mathrm{IU} / \mathrm{L}$ insulin; group 3, $84 \mathrm{mmol} / \mathrm{L}$ glucose and no insulin; group 4, $84 \mathrm{mmol} / \mathrm{L}$ glucose and $10 \mathrm{IU} / \mathrm{L}$ insulin; $L V E F$, left ventricular ejection fraction; $P V D$, peripheral vascular disease.

Table II. Perioperative data

\begin{tabular}{|c|c|c|c|c|c|}
\hline & Group $1(n=10)$ & Group $2(n=12)$ & Group $3(n=17)$ & Group $4(n=17)$ & $\mathrm{P}$ value \\
\hline \multicolumn{6}{|l|}{ Grafts } \\
\hline 3 & $2(20 \%)$ & $0(0 \%)$ & $2(12 \%)$ & $1(6 \%)$ & .13 \\
\hline 4 & $3(30 \%)$ & $6(50 \%)$ & $2(12 \%)$ & $9(53 \%)$ & \\
\hline 5 & $5(50 \%)$ & $6(50 \%)$ & $13(76 \%)$ & $7(41 \%)$ & \\
\hline XCL time (min) & $70 \pm 17$ & $71 \pm 15$ & $75 \pm 16$ & $65 \pm 14$ & .18 \\
\hline CPB time (min) & $87 \pm 18$ & $92 \pm 20$ & $95 \pm 18$ & $85 \pm 14$ & .41 \\
\hline Cardioplegia (L) & $7 \pm 3$ & $7 \pm 2$ & $4 \pm 3$ & $5 \pm 3$ & .89 \\
\hline Highest $\mathrm{K}^{+}$level (mEq/L) & $5.9 \pm 0.5$ & $5.4 \pm 0.6$ & $6.3 \pm 0.5$ & $5.4 \pm 0.7$ & $<.001$ \\
\hline Perioperative MI & $0(0 \%)$ & $1(8 \%)$ & $0(0 \%)$ & $0(0 \%)$ & .31 \\
\hline LOS & $1(10 \%)$ & $1(8 \%)$ & $0(0 \%)$ & $0(0 \%)$ & .36 \\
\hline Length of stay (d) & $7 \pm 1$ & $7 \pm 2$ & $7 \pm 1$ & $6 \pm 1$ & .22 \\
\hline Mortality & $0(0 \%)$ & $0(0 \%)$ & $0(0 \%)$ & $0(0 \%)$ & 1.00 \\
\hline
\end{tabular}

Group 1, $42 \mathrm{mmol} / \mathrm{L}$ glucose and no inulin; group 2, $42 \mathrm{mmol} / \mathrm{L}$ glucose and $10 \mathrm{IU} / \mathrm{L}$ insulin; group 3, $84 \mathrm{mmol} / \mathrm{L}$ glucose and no insulin; group 4, $84 \mathrm{mmol} / \mathrm{L}$ glucose and $10 \mathrm{IU} / \mathrm{L}$ insulin; $X C L$, aortic crossclamp; $C P B$, cardiopulmonary bypass; $M I$, myocardial infarction; LOS, low-output syndrome.

closely reflect current standards of practice and may provide the most potential benefit.

In an earlier rodent study, Kobayashi and Neely ${ }^{12}$ demonstrated that the activity of a key mitochondrial enzyme, pyruvate dehydrogenase (PDH), was inhibited during early reperfusion after global ischemia. More recent studies have demonstrated that the recovery of postischemic myocardial function is dependent on the recovery of PDH activity. ${ }^{13}$ We have recently reported that insulin was capable of stimulating mitochondrial PDH activity in isolated human ventricular cardiomyocytes, which led to improved protection against ischemia and reperfusion. ${ }^{14}$ We performed this prospective, randomized, double-blind trial to determine whether insulin-enhanced cardioplegia conferred similar metabolic or functional benefits to patients undergoing coronary bypass surgery.

\section{Methods}

Patient population. Fifty-six male patients undergoing isolated elective coronary bypass surgery consented to partic- ipate in a metabolic evaluation of insulin-enhanced cardioplegia between September 1995 and July 1997. During this interval, the study surgeon (R.D.W.) performed isolated coronary bypass on 533 patients. A total of 477 patients were excluded because of nonelective surgery $(n=303)$, redo surgery $(n=21)$, or participation in alternative surgical trials $(n=89)$. The remaining patients $(n=64)$ were deemed ineligible to participate because of female sex, diabetes mellitus, recent preoperative infarction, significant left ventricular dysfunction (ejection fraction $<20 \%$ ), or refusal to participate. All patients signed an informed consent form approved by our institutional ethics committee. The preoperative demographics for the study population are given in Table I.

Operative technique. All patients underwent coronary revascularization by one surgeon (R.D.W.). The details of anesthetic induction and intraoperative care have been previously described in detail. ${ }^{15}$ After anesthetic induction, the patient was allocated to 1 of 4 cardioplegic groups determined by means of a computer-generated randomization table (group 1, low glucose and placebo; group 2, low glucose and insulin; group 3, high glucose and placebo; and group 4, high glucose and insulin). Arterial blood was added to crystalloid cardioplegic solution in a $4: 1$ ratio to achieve 


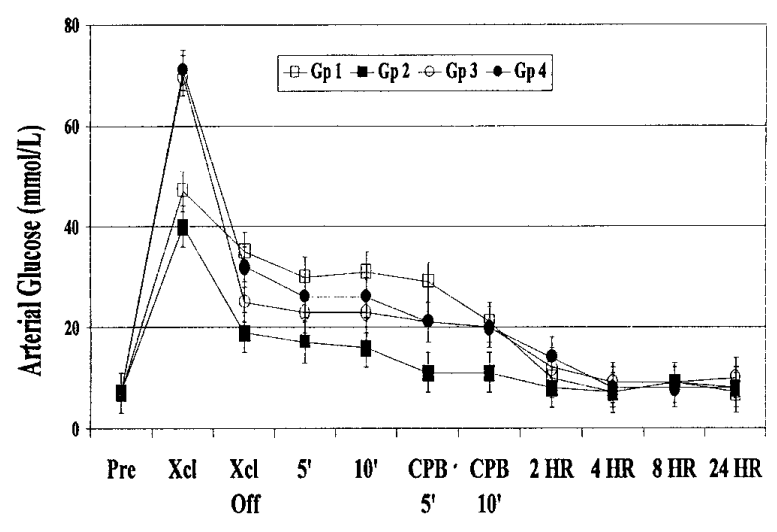

Fig 1. Arterial glucose concentrations in all 4 study groups. The differences in arterial glucose observed during cardioplegic arrest dissipated within 2 hours of arrival into the ICU. There were no overall differences in arterial glucose concentrations between groups. PRE, Prebypass; $\mathrm{Xcl}$, aortic occlusion; Xcl OFF, removal of crossclamp; $C P B$ 10', 10 minutes after discontinuation of cardiopulmonary bypass; 5, 10', 2 $H R, 4 H R, 8 H R, 24 H R$, time after crossclamp removal.

the following final concentrations of all additives. On the basis of our earlier findings and those of other investigators, we chose to administer insulin (10 IU/L Humulin; Eli Lilly Canada, Mississauga, Ontario, Canada) or placebo at two different ambient glucose concentrations ${ }^{9,14}$ : a low-glucose concentration of $42 \mathrm{mmol} / \mathrm{L}$ and a high glucose concentration of $84 \mathrm{mmol} / \mathrm{L}$. In addition to glucose, insulin, or both, all cardioplegic solutions contained equivalent concentrations of potassium chloride $\left(27 \mathrm{mEq} / \mathrm{L}\right.$ for high $\mathrm{K}^{+}$induction and 8 $\mathrm{mEq} / \mathrm{L}$ for low $\mathrm{K}^{+}$maintenance), magnesium sulphate (6 $\mathrm{mEq} / \mathrm{L}$ for all solutions), tris(hydroxymethyl)amino methane (125 mmol/L), and citrate-phosphate-dextrose $(4 \mathrm{mmol} / \mathrm{L})$. The placebo was generously provided by Eli Lilly Canada and contained the nonactive vehicle solution. The clinical perfusionist responsible for the patient was the only individual aware of the randomization group and was responsible for the preparation of all cardioplegic solutions. All other study personnel, including the surgeon, remained blinded to the randomization group.

After institution of cardiopulmonary bypass, all patients received an antegrade induction dose (approximately 1000 $\mathrm{mL})$ of tepid $\left(29^{\circ} \mathrm{C}\right)$ high-potassium blood cardioplegic solution. ${ }^{15}$ After cardioplegic arrest, all patients received nearcontinuous retrograde delivery of cardioplegic solution through the coronary sinus. After completion of each distal anastomosis, the proximal end of the vein graft was attached to a cardioplegic manifold, enabling simultaneous delivery of antegrade and retrograde blood cardioplegic solution. Cardioplegic flow rates were maintained at $200 \mathrm{~mL} / \mathrm{min}$ unless the coronary sinus pressure rose above $40 \mathrm{~mm} \mathrm{Hg}$, whereby the flow rate was adjusted accordingly. ${ }^{16}$ The flow rate remained above $100 \mathrm{~mL} / \mathrm{min}$ in all patients. ${ }^{17}$
Cardioplegic interruptions were limited to less than 7 minutes, and there were no more than 3 interruptions per patient. During cardiopulmonary bypass, the systemic temperature was allowed to drift to $32^{\circ} \mathrm{C}$ to $34^{\circ} \mathrm{C}$. Rewarming to $37^{\circ} \mathrm{C}$ was initiated during construction of the last distal vein graft anastomosis.

To minimize contamination by exogenous insulin delivery, a strict protocol was adopted for the treatment of intraoperative hyperkalemia. An additional cardioplegic solution containing identical additives to the randomization group with the exception of potassium chloride was used initially if the serum potassium level rose above $5 \mathrm{mmol} / \mathrm{L}$ with no evidence of electrical activity. If the potassium level rose above 5.5 $\mathrm{mmol} / \mathrm{L}, 10$ or $20 \mathrm{mg}$ of intravenous furosemide was given to induce diuresis. If the serum potassium concentration rose above $6.0 \mathrm{mmol} / \mathrm{L}$, boluses of human insulin (Humulin; 5-10 IU) were given intravenously. In the event that patients received exogenous insulin or glucose, they remained in their initial randomization group consistent with an intent-to-treat analysis.

After completion of all intraoperative biochemical assessments, the patients were weaned from cardiopulmonary bypass and transferred to the cardiac intensive care unit (ICU). Postoperative ICU care was uniform in all patients and followed an intention to extubate within 6 hours of arrival. ${ }^{18}$ Serial hemodynamic measurements were obtained $2,4,8$, and 24 hours after aortic crossclamp removal, including heart rate, mean arterial pressure, pulmonary capillary wedge pressure, central venous pressure, and cardiac output. Cardiac index, left ventricular stroke work index, and systemic vascular resistance were calculated by using standard formulas. ${ }^{15,16}$

Perioperative myocardial infarction and low cardiac output syndrome were determined by means of previously established criteria. ${ }^{1}$ In brief, the presence of a new Q wave or left bundle branch block on the postoperative electrocardiogram or a significant elevation of creatine kinase MB isoenzyme in the presence of a pre-existing electrocardiographic abnormality was used to define a myocardial infarction. The requirement for intra-aortic balloon or sustained inotropic support for greater than 30 minutes in the ICU defined the development of low cardiac output syndrome. Patients who were given low-dose dopamine $\left(<5 \mu \mathrm{g} \cdot \mathrm{kg}^{-1} \cdot \mathrm{min}^{-1}\right)$ for renal perfusion were not considered to have low-output syndrome.

Biochemical assessments. Arterial and coronary sinus blood samples were obtained at baseline, during each cardioplegic delivery, and at prespecified intervals during reperfusion. In addition, a coronary sinus catheter was left in situ to obtain postoperative blood samples 2, 4, 8, and 24 hours after aortic crossclamp removal. Blood samples were assayed for oxygen content, acid, pyruvate, and lactate concentrations according to previously described protocols. ${ }^{15,16}$ Differences between arterial and coronary sinus blood samples enabled us to calculate myocardial extraction or release of these substances. During cardioplegic arrest, myocardial consumption, or production was calculated after adjusting extractionrelease for coronary flow. 


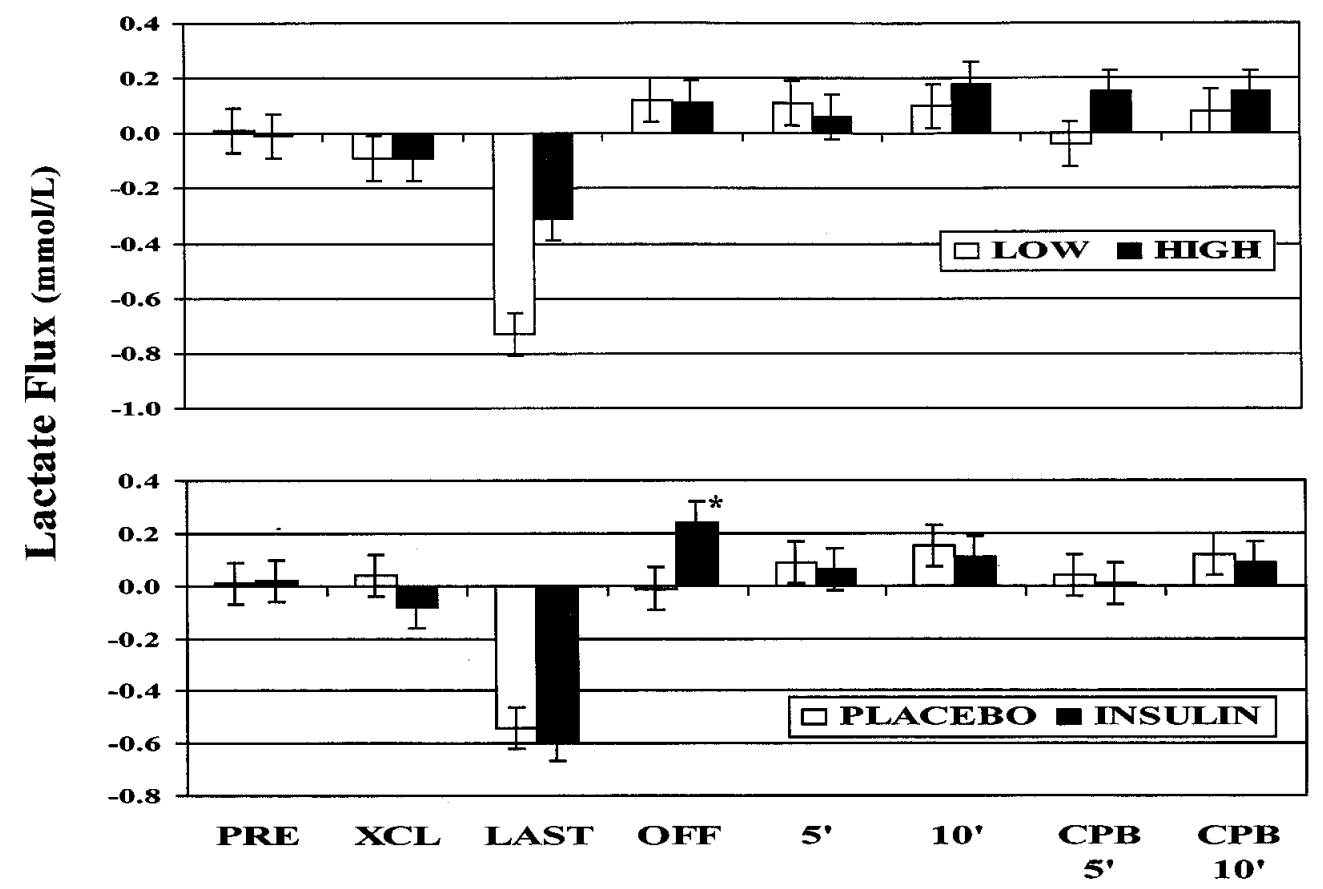

Fig 2. Intraoperative myocardial lactate flux. Cardioplegic arrest induced significant anaerobic lactate release in all patients. Over time, there were no significant effects of either glucose concentration (upper panel, $P=.83$ ) or insulin (lower panel, $P=.28$ ) on myocardial lactate flux. However, immediately after crossclamp removal, patients who received insulin cardioplegia demonstrated lactate extraction compared with persistent anaerobic lactate release in the placebo groups $(P=.03$ by using the Student $t$ test). PRE, Prebypass; $X C L$, aortic occlusion; $O F F$, removal of crossclamp; LAST, final cardioplegic dose.

Left ventricular biopsy specimens were obtained in 8 patients (glucose concentration of $84 \mathrm{mmol} / \mathrm{L}$ with or without insulin; $n=4$ each) for determination of myocardial PDH activity. Full-thickness biopsy specimens were snap-frozen in liquid nitrogen and then homogenized in $250 \mu \mathrm{L}$ of phosphate-buffered saline solution (PBS). Mitochondrial PDH activity was determined by a modification of a previously described technique. ${ }^{14}$ In brief, $75-\mu \mathrm{L}$ aliquots were divided into separate Eppendorf tubes containing $75 \mu \mathrm{L}$ of either PBS or PBS with $5 \mathrm{mmol} / \mathrm{L}$ dichloroacetate. After a 10-minute incubation at $37^{\circ} \mathrm{C}$, the reaction was stopped with a buffer solution containing $25 \mathrm{mmol} / \mathrm{L}$ sodium fluoride to inhibit PDH phosphatase. Next, $50 \mu \mathrm{L}$ from each Eppendorf tube was exposed to $200 \mu \mathrm{L}$ of reaction buffer containing ${ }^{14} \mathrm{C}$ pyruvate. Protein content was simultaneously determined by using a $100-\mu \mathrm{L}$ aliquot from each Eppendorf tube assayed by the Lowry method. ${ }^{14}$ The PDH reaction was then terminated by using $100 \mu \mathrm{L}$ of $10 \%$ trichloroacetic acid. Each Eppendorf tube was then inserted into a scintillation vial containing 200 $\mu \mathrm{L}$ of benzothonium hydroxide. After exposure to each tissue sample for 1 hour, the collected ${ }^{14} \mathrm{CO}_{2}$ was then counted in a $\beta$-counter. PDH activity was calculated after correction for protein content and expressed as nanomoles of pyruvate oxidized per milligram of protein per minute.
Statistical analysis. Statistical analysis was performed by using the SAS analytic program (SAS Institute, Cary, NC). Categoric data were analyzed by using the $\chi^{2}$ or Fisher exact test as appropriate. Continuous data were analyzed by using analysis of variance and are expressed as mean \pm SD, unless otherwise specified. Two-way repeated-measures analysis of variance was used to analyze biochemical end points over time. To correct for patient-specific activities of PDH, the percentage recovery of PDH activity was calculated for each patient, and the effects of time and group were assessed with analysis of covariance. Hemodynamic data were analyzed by means of analysis of covariance, examining the main effects of time, group, and preload. Left ventricular preload was estimated from measurements of pulmonary capillary wedge pressure. Exact $P$ values are reported to enable determination of clinical and statistical significance.

\section{Results}

Operative data. Table II summarizes the perioperative data for all 4 randomization groups. Randomization groups were similar with respect to the number of distal anastomoses performed, the mean aortic crossclamp and cardiopulmonary bypass times, and the volume of car- 


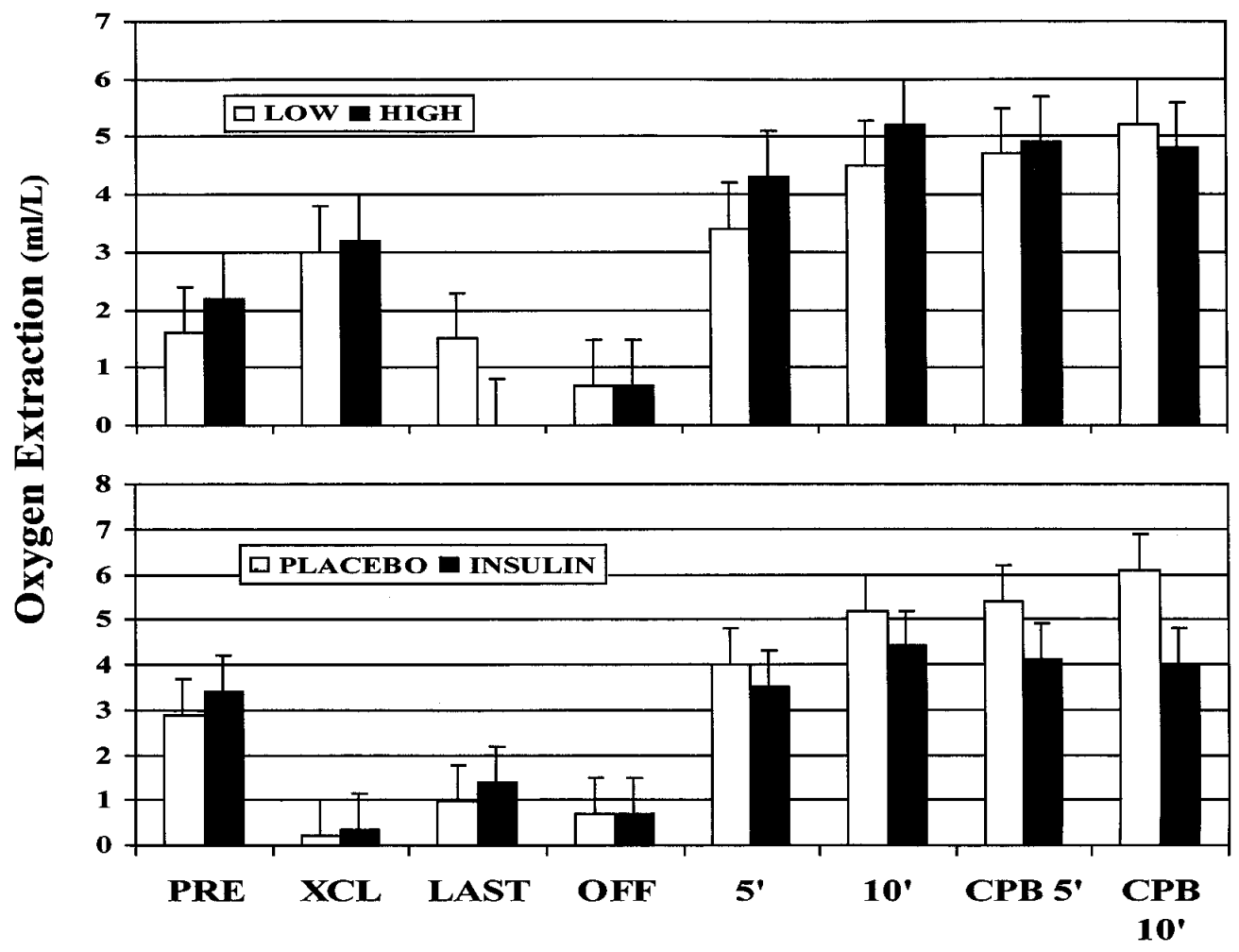

Fig 3. Effects of glucose (upper panel) and insulin (lower panel) on intraoperative myocardial oxygen extraction. During the crossclamp period, there was a significant interactive effect between glucose and insulin $(P=.03)$. During reperfusion, myocardial oxygen extraction returned to baseline in the insulin cardioplegia groups. However, there was increased oxygen extraction in the placebo groups, suggestive of repayment of an oxygen debt. There was no significant effect of glucose concentration $(P=.33)$; however, there was a significant insulin effect over time (insulin-time effect, $P=.04$ ). $P R E$, Prebypass; $X C L$, aortic occlusion; $O F F$, removal of crossclamp; LAST, final cardioplegic dose.

dioplegic solution delivered. There were no differences in the times of cardioplegic interruption expressed as a percentage of the total crossclamp time $(P=.6)$. However, patients exposed to insulin cardioplegia at either ambient glucose concentration had a lower peak serum potassium level than patients in groups 1 and $3(P<.001)$. Persistent hyperkalemia required insulin boluses in 6 patients (11\%), 5 who received placebo and 1 who received insulin cardioplegia $(P=.07)$. Hypoglycemia requiring supplemental dextrose administration occurred in 5 patients, 3 of whom received insulin and high-glucose cardioplegia. The remaining 2 patients received low-glucose cardioplegia with placebo. Interestingly, there was no observed hypoglycemia in the low-glucose (42 $\mathrm{mmol} / \mathrm{L}$ ) and insulin groups. Fig 1 illustrates arterial glucose levels, which were similar in all 4 study groups during the experimental protocol.

There was no mortality in this study population. There was 1 patient in group 2 who had a perioperative myocardial infarction and had postoperative low cardiac output syndrome. One additional patient in group 1 had low-output syndrome without evidence of infarction. The postoperative length of stay was similar in all 4 groups. No permanent neurologic deficits were observed in any patients, including the 5 patients who had hypoglycemia.

Biochemical outcomes. Fig 2 demonstrates the effects of glucose (upper panel) and insulin (lower panel) on myocardial lactate release during the surgical procedure. There were no significant effects of glucose $(P=.8)$ or insulin $(P=.3)$ over time. During the crossclamp period, there were no differences between groups in myocardial lactate production (corrected for coronary blood flow). However, at crossclamp removal, patients who received insulin-enhanced cardioplegic solution displayed a positive lactate flux ( $P=.03$ by the Student $t$ test) compared with a negative flux in those who received placebo, suggesting better aerobic metabolism. 


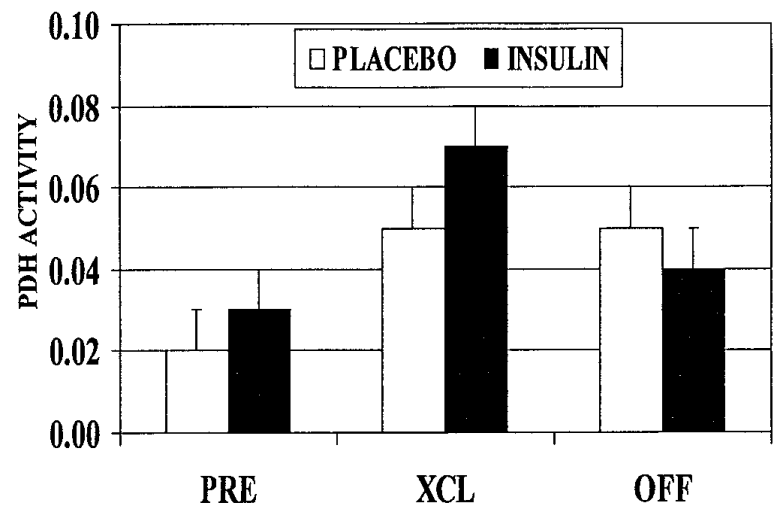

Fig 4. Myocardial PDH activity (nanomoles per milligrams per minute) before cardioplegic arrest (PRE), just before aortic crossclamp removal $(X C L)$, and after 10 minutes of reperfusion $(O F F)$. PDH activity increased during cardioplegic arrest but returned to baseline values during early reperfusion (time effect, $P=.009$ ). There were no significant effects of either glucose or insulin on PDH activity (group effect, $P=.48$ ).

Fig 3 demonstrates the effects of glucose (upper panel) and insulin (lower panel) on myocardial oxygen extraction during the surgical procedure. During the crossclamp period, there was a significant interactive effect between glucose and insulin $(P=.03)$. When corrected for coronary blood flow, myocardial oxygen consumption was affected by both ambient glucose concentration and insulin (glucose $\cdot$ insulin effect, $P=$ .07). During reperfusion, myocardial oxygen extraction returned to baseline in the insulin cardioplegia groups. However, there was increased oxygen extraction in the placebo groups, suggestive of repayment of an oxygen debt. There was no significant effect of glucose concentration $(P=.33)$; however, there was a significant insulin effect over time (insulin - time effect, $P=.04$ ).

Fig 4 demonstrates the activity of mitochondrial PDH measured just before cardioplegic arrest, at the end of the crossclamp period, and after 10 minutes of reperfusion. There was a significant effect of cardioplegic arrest on myocardial PDH activity (time effect, $P=$ .009). During the crossclamp period, PDH activity rose to near maximal levels; however, this activation was attenuated during early reperfusion. We were unable to demonstrate an insulin effect on myocardial PDH activity at any time point (group effect, $P=.5$ )

Hemodynamic outcomes. Table III provides the raw hemodynamic data for all 4 groups. Fig 5 demonstrates the improvement in left ventricular performance seen in the insulin cardioplegia groups 2 hours after surgery. Both cardiac index and left ventricular stroke work index were greater in the insulin cardioplegia groups at
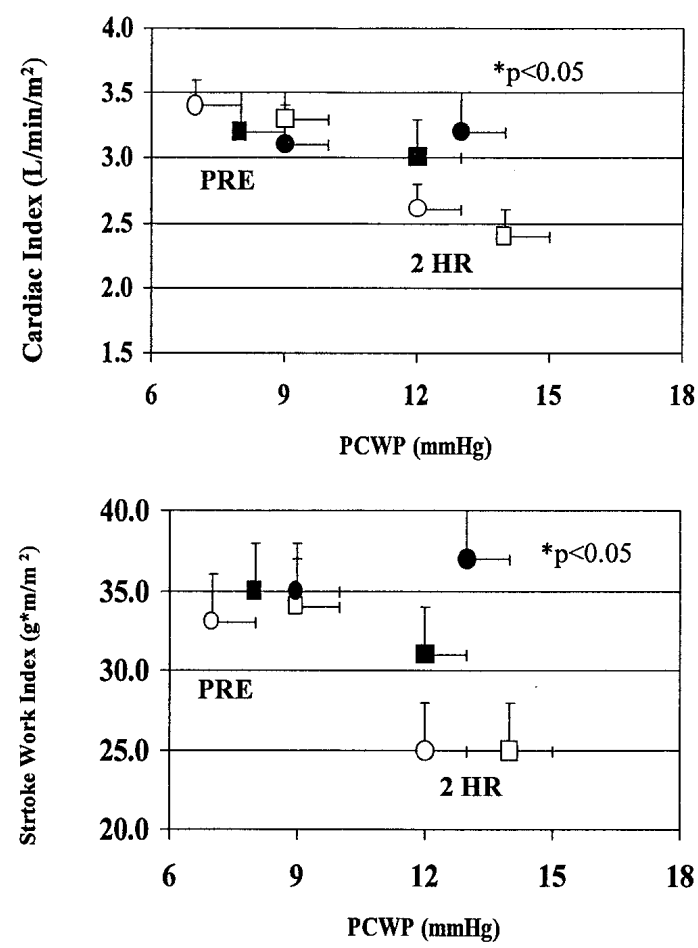

Fig 5. Cardiac index (upper panel) and left ventricular stroke work index (lower panel) at 2 hours after surgery. Patients who received insulin cardioplegia demonstrated enhanced left ventricular performance compared with placebo at similar filling pressures. $P C W P$, Pulmonary capillary wedge pressure. Circles represent low-glucose cardioplegia, squares represent high-glucose cardioplegia, open symbols represent placebo groups, and closed symbols represent insulin groups.

similar filling pressures. Insulin-enhanced cardioplegia induced a significant vasodilatory effect, as evidenced by the lower systemic vascular resistance compared with the placebo groups.

\section{Discussion}

Coronary bypass surgery continues to be associated with low morbidity and mortality despite an increasing proportion of high-risk patients. ${ }^{4}$ Although a wide variety of myocardial protective strategies may be successful in low-risk patients with preserved left ventricular function, cardioplegic additives may be required for those patients who have acute ischemic syndromes or impaired left ventricular function. We have previously demonstrated that cardioplegic interventions that yield metabolic and functional benefits in low-risk patients produce improvements in morbidity and mortality in high-risk subgroups. ${ }^{19} \mathrm{We}$ designed this prospective randomized study to determine the metabolic and functional effects of glucose and insulin in low-risk patients undergoing isolated coronary 
Table III. Postoperative hemodynamic data

\begin{tabular}{|c|c|c|c|c|c|}
\hline & Group $1(n=10)$ & Group $2(n=12)$ & Group $3(n=17)$ & Group $4(n=17)$ & $\mathrm{P}$ value \\
\hline \multicolumn{6}{|l|}{$2 \mathrm{~h}$} \\
\hline Heart rate (beats/min) & $80 \pm 12$ & $82 \pm 12$ & $85 \pm 19$ & $89 \pm 22$ & .71 \\
\hline PCWP (mm Hg) & $14 \pm 2$ & $12 \pm 1$ & $12 \pm 1$ & $13 \pm 1$ & .53 \\
\hline Cardiac index (L/min) & $2.4 \pm 0.2$ & $3.0 \pm 0.1$ & $2.6 \pm 0.2$ & $3.2 \pm 0.3$ & .01 \\
\hline $\operatorname{LVSWI}\left(\mathrm{g} \cdot \mathrm{m}^{-1} \cdot \mathrm{m}^{-2}\right)$ & $25 \pm 2$ & $31 \pm 2$ & $25 \pm 2$ & $31 \pm 2$ & .08 \\
\hline SVRI & $2571 \pm 224$ & $1790 \pm 102$ & $1962 \pm 228$ & $1715 \pm 165$ & .01 \\
\hline \multicolumn{6}{|l|}{$4 \mathrm{~h}$} \\
\hline Heart rate (beats/min) & $90 \pm 10$ & $78 \pm 12$ & $87 \pm 19$ & $84 \pm 18$ & .26 \\
\hline PCWP (mm Hg) & $17 \pm 2$ & $15 \pm 1$ & $14 \pm 2$ & $15 \pm 1$ & .61 \\
\hline Cardiac index (L/min) & $2.4 \pm 0.1$ & $2.5 \pm 0.1$ & $3.0 \pm 0.2$ & $2.8 \pm 0.2$ & .07 \\
\hline $\operatorname{LVSWI}\left(\mathrm{g} \cdot \mathrm{m}^{-1} \cdot \mathrm{m}^{-2}\right)$ & $24 \pm 3$ & $26 \pm 2$ & $29 \pm 1$ & $28 \pm 3$ & .34 \\
\hline SVRI & $2444 \pm 165$ & $2053 \pm 128$ & $1913 \pm 217$ & $1922 \pm 186$ & .10 \\
\hline \multicolumn{6}{|l|}{$8 \mathrm{~h}$} \\
\hline Heart rate (beats/min) & $86 \pm 13$ & $84 \pm 15$ & $90 \pm 12$ & $86 \pm 15$ & .84 \\
\hline PCWP (mm Hg) & $16 \pm 2$ & $13 \pm 1$ & $14 \pm 1$ & $12 \pm 2$ & .38 \\
\hline Cardiac index (L/min) & $2.7 \pm 0.1$ & $2.8 \pm 0.2$ & $2.7 \pm 0.2$ & $2.7 \pm 0.2$ & .81 \\
\hline $\operatorname{LVSWI}\left(\mathrm{g} \cdot \mathrm{m}^{-1} \cdot \mathrm{m}^{-2}\right)$ & $26 \pm 3$ & $29 \pm 2$ & $30 \pm 2$ & $25 \pm 1$ & .44 \\
\hline SVRI & $2001 \pm 174$ & $1982 \pm 102$ & $2507 \pm 292$ & $1920 \pm 129$ & .14 \\
\hline \multicolumn{6}{|l|}{$24 \mathrm{~h}$} \\
\hline Heart rate (beats/min) & $90 \pm 11$ & $79 \pm 13$ & $85 \pm 15$ & $86 \pm 17$ & .31 \\
\hline PCWP (mm Hg) & $13 \pm 2$ & $11 \pm 1$ & $13 \pm 2$ & $13 \pm 2$ & .64 \\
\hline Cardiac index (L/min) & $2.8 \pm 0.2$ & $2.8 \pm 0.2$ & $2.6 \pm 0.1$ & $2.7 \pm 0.1$ & .70 \\
\hline $\operatorname{LVSWI}\left(\mathrm{g} \cdot \mathrm{m}^{-1} \cdot \mathrm{m}^{-2}\right)$ & $31 \pm 3$ & $31 \pm 3$ & $27 \pm 2$ & $29 \pm 2$ & .75 \\
\hline SVRI & $2301 \pm 176$ & $1922 \pm 90$ & $2069 \pm 88$ & $2108 \pm 137$ & .22 \\
\hline
\end{tabular}

Group 1, $42 \mathrm{mmol} / \mathrm{L}$ glucose and no insulin; group 2, $42 \mathrm{mmol} / \mathrm{L}$ glucose and $10 \mathrm{IU} / \mathrm{L}$ insulin; group 3, $84 \mathrm{mmol} / \mathrm{L}$ glucose and no insulin; group 4, $84 \mathrm{mmol} / \mathrm{L}$ glucose and $10 \mathrm{IU} / \mathrm{L}$ insulin; PCWP, Pulmonary capillary wedge pressure; LVSWI, left ventricular stroke work index; SVRI, systemic vascular resistance index.

bypass surgery with a strategy of tepid antegrade-retrograde blood cardioplegia.

Biochemical response to glucose and insulin. Patients in groups 2 and 4 (insulin-enhanced cardioplegia) received approximately $60 \mathrm{U}$ of short-acting human insulin during 1 hour of cardioplegic arrest. Despite this large dose of insulin, there were no significant differences in arterial glucose concentrations in the early postoperative period. This is likely because of the short half-life of insulin in blood combined with our approach of direct myocardial delivery. Adding insulin to the crystalloid component of the cardioplegic solution resulted in a first-pass effect, whereby insulin was directly exposed to myocardial receptors before venous drainage from the right atrium. We believe that this mode of delivery is inherently safer than systemic delivery through a central vein. Only 5 patients required supplemental glucose, and of these, only 2 had severe hypoglycemia $(<3 \mathrm{mmol} / \mathrm{L})$. There were no adverse neurologic outcomes in any of the 4 study groups.

Our previous cellular studies indicated that insulin was capable of stimulating myocardial PDH activity. ${ }^{14}$ The theoretic benefit of PDH stimulation involves an earlier transition from anaerobic to aerobic metabolism after an ischemic insult. Weiss and Hiltbrand ${ }^{20}$ reported that adenosine triphosphate produced by oxidative phosphorylation was preferentially used for contractile function compared with anaerobically produced adenosine triphosphate, which was used for membrane stability. ${ }^{20}$ Furthermore, Lewandowski and White ${ }^{13}$ demonstrated that postischemic left ventricular function was dependent on the recovery of PDH activity. Unfortunately, we were unable to detect an insulin effect on the activity of myocardial PDH. Although cardioplegic arrest and reperfusion greatly influenced the activity of PDH, our small sample size did not allow us to detect a significant stimulatory effect of insulin. However, myocardial oxygen consumption was higher during cardioplegic arrest in the insulin groups, suggesting enhanced aerobic activity. Although the placebo group demonstrated repayment of an oxygen debt during early reperfusion, myocardial oxygen extraction was similar to baseline in the insulin cardioplegia groups. Although there appeared to be an insulin effect during cardioplegic arrest, the differences in myocardial PDH activity failed to achieve statistical significance, probably because of the small number of patients who had biopsies. Myocardial lactate flux was not affected by cardioplegia group, although at one 
time point (crossclamp removal) the insulin-enhanced groups demonstrated greater lactate flux, again suggestive of improved aerobic capacity. In previous cell culture studies, we found that insulin resulted in higher $\mathrm{PDH}$ activity after 30 minutes of reperfusion. ${ }^{14}$ In this protocol we assessed PDH activity at 10 minutes of reperfusion. Similar to the results obtained from Kobayashi and Neely, ${ }^{12}$ we found an inhibition of PDH activity in the early postischemic period. We hypothesize that the improvements in left ventricular performance observed in the insulin cardioplegia groups are a result of enhanced myocardial PDH activity at 2 hours of reperfusion.

Hemodynamic effects. Although we were unable to detect major biochemical differences between groups, we did demonstrate a significant hemodynamic benefit in the insulin cardioplegia groups. Both cardiac index and stroke work index were higher in the insulin cardioplegia groups at similar filling pressures. This hemodynamic benefit was evident 2 hours after surgery but dissipated by the eighth postoperative hour. There was a marked reduction in systemic vascular resistance in the insulin cardioplegia group, an effect previously reported by other investigators. ${ }^{21}$ The combination of increased inotropy with decreased afterload may prove to be most efficacious in patients with poor ventricular function. We are presently completing a clinical trial to evaluate the salutatory effects of insulin in this highrisk population.

\section{Summary}

This prospective study was designed to evaluate the effects of insulin and glucose as cardioplegic additives on the recovery of myocardial metabolism and function after elective coronary bypass surgery. Insulin-enhanced cardioplegia demonstrated both metabolic and functional benefit independent of the ambient glucose concentration. Because of the possible deleterious effects of hyperglycemia on postoperative neurologic outcomes, ${ }^{22}$ we recommend a low-glucose cardioplegic formulation, even when insulin is used as an additive. The potential clinical benefits of insulin-enhanced cardioplegia will require further investigation in high-risk subgroups of patients undergoing myocardial revascularization.

We thank the clinical perfusionists and the nursing staff of the Cardiovascular Intensive Unit of the Toronto General Hospital for their assistance in completing this study.

\section{REFERENCES}

1. Rao V, Ivanov J, Weisel RD, Ikonomidis JS, Christakis GT, David TE. Predictors of low output syndrome after coronary artery bypass. J Thorac Cardiovasc Surg 1996;112:38-51.
2. Hamm CW, Reimers J, Ischinger T, et al. A randomized study of coronary angioplasty versus bypass-surgery in patients with symptomatic multivessel coronary disease. N Engl J Med 1994; 331:1037-43.

3. Calafiore AM, Di Giammarco G, Teodori G, et al. Midterm results after minimally invasive coronary surgery (LAST operation). J Thorac Cardiovasc Surg 1998;115:763-71.

4. Christakis GT, Ivanov J, Weisel RD, et al. The changing patterns of coronary artery bypass surgery. Circulation 1988;80:(Suppl):I51-61.

5. Rao V, Christakis GT, Weisel RD, et al. Changing pattern of valve surgery. Circulation 1996;94(Suppl):II-113-20.

6. Christakis GT, Weisel RD, Fremes SE, et al. Coronary artery bypass surgery in patients with poor ventricular function. J Thorac Cardiovasc Surg 1992;103:1083-92.

7. Christakis GT, Fremes SE, Weisel RD, et al. Reducing the risk of urgent revascularization for unstable angina: randomized clinical trial. J Vasc Surg 1986;3:764-72.

8. Ivanov J, Weisel RD, David TE, Naylor CD. Fifteen-year trends in risk severity and operative mortality in elderly patients undergoing coronary artery bypass grafting. Circulation 1998;97:673-80.

9. Lazar HL. Enhanced preservation of acutely ischemic myocardium using glucose-insulin-potassium solutions. J Card Surg 1994; 9(Suppl):474-8.

10. Rao V, Cohen G, Weisel RD, et al. The use of glucose and insulin during hypothermic and normothermic CABG. Ann N Y Acad Sci 1996;793:494-7.

11. Lichtenstein SV, Ashe KA, El Dalati H, Cusimano RJ, Panos A, Slutsky AS. Warm heart surgery. J Thorac Cardiovasc Surg 1991; 101:269-74.

12. Kobayashi K, Neely JR. Effects of ischemia and reperfusion on pyruvate dehydrogenase activity in isolated rat hearts. J Mol Cell Cardiol 1983;15:359-67.

13. Lewandowski ED, White LT. Pyruvate dehydrogenase influences postischemic heart function. Circulation 1995;91:2071-9.

14. Rao V, Merante F, Weisel RD, et al. Insulin stimulates pyruvate dehydrogenase and protects human ventricular cardiomyocytes from simulated ischemia. J Thorac Cardiovasc Surg 1998;116: 485-94.

15. Hayashida N, Weisel RD, Shirai T, et al. Tepid antegrade and retrograde cardioplegia. Ann Thorac Surg 1995;59:723-9.

16. Shirai T, Rao V, Weisel RD, et al. Antegrade and retrograde cardioplegia: Alternate or simultaneous? J Thorac Cardiovasc Surg 1996;112:787-96.

17. Rao V, Cohen G, Weisel RD, et al. Optimal flow rates for integrated cardioplegia. J Thorac Cardiovasc Surg 1998;115:226-35.

18. Cheng DCH, Karski J, Peniston C, et al. Morbidity outcome of early versus conventional tracheal extubation after coronary artery bypass graft surgery: a prospective, randomized, controlled clinical study. J Thorac Cardiovasc Surg 1996;112:755-64.

19. Ikonomidis JS, Rao V, Weisel RD, Hayashida N, Shirai T. Myocardial protection for coronary bypass surgery: the Toronto Hospital perspective. Ann Thorac Surg 1995;60:824-32.

20. Weiss J, Hiltbrand B. Functional compartmentation of glycolytic versus oxidative metabolism in isolated rabbit heart. J Clin Invest 1985;75:436-47.

21. Svensson S, Berglin E, Ekroth R, Milocco I, Nilsson F, WilliamOlson G. Haemodynamic effects of a single large-dose of insulin in open heart surgery. Cardiovasc Res 1984;18:697-701.

22. Martin TD, Craver JM, Gott JP, et al. Prospective randomized trial of retrograde warm blood cardioplegia: myocardial benefit and neurologic threat. Ann Thorac Surg 1994;57:298-304. 


\section{Appendix}

Insulin Cardioplegia Trial (ICT) investigators

Steering Committee. George T. Christakis, MD; Joan Ivanov RN, MSc; C. David Naylor, MD, DPhil; Vivek Rao, MD, PhD; Richard D. Weisel, MD.

Trial Coordinators. Michael A. Borger, MD; Susan M. Carson, AHT; Gideon Cohen, MD; Vania DeSouza, MD; Barbara Weller, RN.

Safety and Outcomes Committee. Davey Cheng, MD; Jacek Karski, MD; Keyvan Kartoukian, MD; C. David Mazer, MD; Terry M. Smith, MD; Bill I. Wong, MD; Terrence M. Yau, MD. Biochemical Support. Frank Merante, PhD; Donald A. G. Mickle, MD; Molly K. Mohabeer; Laura C. Tumiati.

Participating Surgeons. Gopal Bhatnagar, MD; Daniel Bonneau, MD; George T. Christakis, MD; Robert J. Cusimano, MD; Tirone E. David, MD; Lee M. Errett, MD; Christopher M. Feindel, MD; Stephen E. Fremes, MD; Bernard S. Goldman, MD; Lynda L. Mickleborough, MD; Charles M. Peniston, MD; Anthony Ralph-Edwards, MD; Hugh E. Scully, MD; Glen S. Van Arsdell, MD; Richard D. Weisel, MD; Terrence M. Yau, MD.

\section{Commentary}

Rao and associates are to be congratulated for carrying out a rare feat in cardiac surgery, a prospective randomized trial comparing two surgical therapeutic options. This randomized trial of 56 patients undergoing coronary bypass grafting with continuous tepid cardioplegia demonstrated that the addition of insulin in the cardioplegic solution decreased initial systemic vascular resistance, increased early cardiac output and stroke work, decreased serum potassium levels, and improved early cardiac lactate extraction. The authors concluded that insulin cardioplegia improved myocardial metabolic and functional recovery.

The reasons for few randomized surgical trials are many. When cardiac function is the end point, detecting subtle differences in cardiac function can be very difficult during concurrent changes in cardiac load. In this study the authors were fortunate that the treatment group had lower cardiac afterload and similar cardiac preload, while having increased stroke work. Had afterload increased, it might have been difficult to determine whether increased stroke work was caused by increased contractility or by increased afterload.

Like any good study, this one raises several questions that remain to be answered:

1. Do the beneficial effects of insulin cardioplegia in continuous tepid cardioplegia also apply to intermittent cold cardioplegia? Today, intermittent cold cardioplegia is far more commonly used than continuous tepid cardioplegia. The authors suggest that insulin may in fact not be beneficial in intermittent cold cardioplegia. Previous research looking at glucose-insulin-potassium cardioplegia never conclusively found benefit in cold intermittent cardioplegia. The reason may be, as the authors suggest, that insulin may overcome a block in mitochondrial glucose metabolism at the level of pyruvate dehydrogenase at warmer temperatures, although not at colder temperatures where enzyme activities may be markedly different. If so, the value of this study will await greater evidence concerning the practicality and benefits of warm tepid cardioplegia over intermittent cold cardioplegia.

2. Given the relatively short 2-hour duration of benefits realized from insulin cardioplegia in this study, are these benefits great enough to be of any consequence? Perhaps the benefit may be greater in the small number of cases in which myocardial protection may still be suboptimal with current techniques (ie, those with longer ischemic times [especially cardiac transplantation] or those with poor baseline ventricular function). The applicability of continuous tepid cardioplegia to longer ischemic times needs to be demonstrated, given that metabolic demands will also be greater at tepid temperatures than at colder temperatures. Are the total potassium loads of continuous tepid cardioplegia over longer ischemic times adequately tolerated by patients, particularly in patients with baseline renal insufficiency?

3 . As in point 1 above, what precisely is the mechanism by which insulin benefits the heart in continuous tepid cardioplegia? The answer to this may help to clarify the role of insulin in cold intermittent cardiology.

This study by Rao and associates will certainly need to be confirmed by other investigators in other settings before insulin can be considered a standard additive to continuous tepid cardioplegia. In the interim, the relatively clear and positive results of this study should encourage other cardiac surgical investigators to consider prospective randomized trials. Studies like this could avoid years of heated debate in interpreting large amounts of data that are more easily obtained but of much lower quality.

Donald D. Glower, MD Durham, North Carolina 12/1/105832 doi:10.1067/mtc.2000.105832 